\title{
One-loop renormalisation of lattice QCD operators for non-forward matrix elements: from clover to overlap fermions
}

\author{
QCDSF collaboration: M. Göckeler, ${ }^{a}$ R. Horsley, ${ }^{b}$ H. Perlt,${ }^{c}$ P. E. L. Rakow, ${ }^{d}$ \\ G. Schierholz, ${ }^{e}$ A. Schiller ${ }^{c}$ A. Schiller* \\ ${ }^{a}$ Institut für Theoretische Physik, Universität Regensburg, 93040 Regensburg, Germany \\ ${ }^{b}$ School of Physics, University of Edinburgh, Edinburgh EH9 3JZ, UK \\ ${ }^{c}$ Institut für Theoretische Physik, Universität Leipzig, 04109 Leipzig, Germany \\ ${ }^{d}$ Theoretical Physics Division, Department of Mathematical Sciences, University of Liverpool, \\ Liverpool L69 3BX, UK \\ e John von Neumann Institut NIC/DESY Zeuthen, 15738 Zeuthen Germany, \\ Deutsches Elektronen-Synchrotron DESY, 22603 Hamburg, Germany
}

\begin{abstract}
We consider the renormalisation of composite quark-antiquark operators with one and two lattice covariant derivatives related to the lowest moments of generalised parton distributions (GPDs) and meson distribution amplitudes (DAs). Their matrix elements are calculated in one-loop lattice perturbation theory for non-zero momentum transfer. Using clover and overlap fermions we present the resulting matrices of mixing and renormalisation factors. For overlap fermions we explicitly check the absence of mixing with lower-dimensional operators of different chirality in particular representations of the hypercubic group. This feature favours the use of chiral fermions.
\end{abstract}

XXIVth International Symposium on Lattice Field Theory

July 23-28, 2006

Tucson, Arizona, USA

\footnotetext{
*Speaker.
} 


\section{Introduction}

Many interesting observables in hadron physics, e.g. (moments of) generalised parton distributions (GPDs) or distribution amplitudes (DAs), can be computed from matrix elements of local operators between hadron states. (For an extensive review of GPDs see Ref. [1], for DAs see, e.g., Ref. [2]].) GPDs parametrise a large class of hadronic correlators, including form factors and the ordinary parton distribution functions. Thus those distributions provide a formal framework to connect information from various inclusive, semi-inclusive and exclusive reactions. Furthermore they give access to physical quantities which cannot be directly determined in experiments, like e.g. the orbital angular momentum of quarks and gluons in a nucleon (in a given scheme) and the spatial distribution of the energy or spin density of a fast moving hadron in the transverse plane. Since the structure of GPDs is rather complicated direct experimental access is limited. Therefore, complementary information from lattice QCD should be used. For a recent overview on Monte Carlo results, see Ref. [3].

To make contact with the continuum, the lattice operators needed for GPDs and DAs have to be renormalised. Compared with moments of ordinary parton distributions, the specific difficulty in the treatment of moments of GPDs and DAs lies in the fact that the required matrix elements are no longer forward matrix elements. This circumstance complicates the pattern of mixing under renormalisation.

In Ref. [⿰亻 malisation of quark-antiquark operators with two derivatives, which determine the second moments of GPDs, and we have discussed the mixing problem in detail. This calculation was performed in one-loop lattice perturbation theory for the Wilson fermion action.

Here we present some of our results [5] obtained for $O(a)$ improved fermions using the Sheikholeslami-Wohlert (clover) action [6] (without operator improvement) and new calculations for overlap fermions [7].

The fermion action is of the following generic form (spinor and colour indices are suppressed)

$$
S_{F}=\bar{\psi} D \psi \equiv a^{4} \sum_{x, y} \bar{\psi}(x) D(x-y) \psi(y)
$$

with

$$
\begin{aligned}
D_{S W} & =D_{W}-\mathrm{i} \operatorname{agr} c_{S w} \frac{1}{8}\left[\gamma_{\mu}, \gamma_{\nu}\right] F_{\mu \nu}^{\text {clover }}, \\
D_{N} & ==\frac{\rho}{a}\left(1+\frac{X}{\sqrt{X^{\dagger} X}}\right), \quad X=D_{W}-\frac{\rho}{a}
\end{aligned}
$$

for the clover $\left(D_{S W}\right)$ and overlap $\left(D_{N}\right)$ operator with massless quarks, respectively. Here $D_{W}$ denotes the Wilson-Dirac fermion operator with the forward (backward) covariant derivative $\Delta_{\mu}\left(\Delta_{\mu}^{*}\right)$

$$
D_{W}=\frac{1}{2}\left[\gamma_{\mu}\left(\Delta_{\mu}^{*}+\Delta_{\mu}\right)-\operatorname{ar} \Delta_{\mu}^{*} \Delta_{\mu}\right], \quad \Delta_{\mu} \psi(x)=\frac{1}{a}\left[U_{x, \mu} \psi(x+a \hat{\mu})-\psi(x)\right] .
$$

$g$ is the bare coupling, $a$ the lattice spacing and $F_{\mu \nu}^{\text {clover }}$ the standard "clover-leaf" form of the lattice field strength

$$
F_{\mu \nu}^{\text {clover }}=\frac{1}{8 \mathrm{i} g a^{2}} \sum_{\mu, v= \pm}\left(U_{x, \mu v}^{\square}-U_{x, \mu \nu}^{\square \dagger}\right) .
$$


In the perturbative calculation the operators to be investigated are sandwiched between offshell quark states with 4-momenta $p$ and $p^{\prime}$. Our calculations are performed in a general covariant gauge, in both cases the final numbers are presented in Feynman gauge for the Wilson parameter $r=1$. The clover results are presented with the improvement term $c_{s w}$ left free, the overlap results are found for overlap parameter $\rho=1.4$.

In [4] a detailed discussion of the renormalisation procedure for the case of non-vanishing momentum transfer can be found. The matrix of renormalisation and mixing coefficients $Z_{i k}(a \mu)$ relating regularised lattice vertex functions $\Gamma_{k}^{L}\left(p^{\prime}, p, a, g_{\mathrm{R}}\right)$ and $\overline{\mathrm{MS}}$ renormalised vertex functions $\Gamma_{i}^{R}\left(p^{\prime}, p, \mu, g_{\mathrm{R}}\right)$ is defined such that

$$
\Gamma_{i}^{R}\left(p^{\prime}, p, \mu, g_{\mathrm{R}}\right)=Z_{\psi}^{-1}(a \mu) \sum_{k=1}^{N} Z_{i k}(a \mu) \Gamma_{k}^{L}\left(p^{\prime}, p, a, g_{\mathrm{R}}\right)
$$

with the quark wave function renormalisation constant $Z_{\psi}$. Here $p\left(p^{\prime}\right)$ denotes the momentum of the incoming (outgoing) quark, the renormalisation scale is $\mu$, the renormalised coupling is denoted by $g_{\mathrm{R}}$, and $N$ is the number of operators which mix in the one-loop approximation.

\section{Operators and mixing}

We consider quark-antiquark operators with up to two covariant symmetric lattice derivatives $\overleftrightarrow{D}=\vec{D}-\overleftarrow{D}$ (for their definitions, see e.g. [[8]) and external ordinary derivatives $\partial$

In the non-forward case $q=p^{\prime}-p \neq 0$ we use two realisations of operators with covariant derivatives in momentum space: either the momentum transfer "acts" at the position $x$ associated with the operator (realisation I) or $q$ is applied at the point half way between the quark fields $\bar{\psi}$ and $\psi$ (II). As an example, for two covariant derivatives this leads to

$$
\begin{aligned}
\left(\bar{\psi} \stackrel{\leftrightarrow}{D}_{\mu} \overleftrightarrow{D}_{v} \psi\right)^{(I I)}(q)=\frac{1}{a^{2}} \sum_{x}\left(\bar{\psi}(x) U_{x, \mu} U_{x+a \hat{\mu}, v} \psi(x+a \hat{\mu}+a \hat{v})\right. \\
-\bar{\psi}(x+a \hat{v}) U_{x+a \hat{v}, \mu} U_{x+a \hat{\mu}, v}^{\dagger} \psi(x+a \hat{\mu}) \\
-\bar{\psi}(x+a \hat{\mu}) U_{x, \mu}^{\dagger} U_{x, v} \psi(x+a \hat{v}) \\
\left.+\bar{\psi}(x+a \hat{\mu}+a \hat{v}) U_{x+a \hat{v}, \mu}^{\dagger} U_{x, v}^{\dagger} \psi(x)\right) \mathrm{e}^{\mathrm{i} q \cdot(x+a \hat{\mu} / 2+a \hat{v} / 2)}
\end{aligned}
$$

and realisation I is obtained from (2.1) as

$$
\left(\bar{\psi} \stackrel{\leftrightarrow}{D}_{\mu} \stackrel{\leftrightarrow}{D}_{\nu} \psi\right)^{(I)}(q)=\cos \left(\frac{a q_{\mu}}{2}\right) \cos \left(\frac{a q_{v}}{2}\right)\left(\bar{\psi} \stackrel{\leftrightarrow}{D}_{\mu} \stackrel{\leftrightarrow}{D}_{v} \psi\right)^{(I I)}(q)
$$

We have set the Dirac matrix in the operators equal to the unit matrix for simplicity.

In what follows, we indicate in the operator symbols the derivatives by superscripts $D$ and $\partial$. The quark-antiquark operators with one derivative are given by

$$
\begin{gathered}
\mathscr{O}_{\mu \nu}^{D}=-\frac{\mathrm{i}}{2} \bar{\psi} \gamma_{\mu} \stackrel{\leftrightarrow}{D}_{\nu} \psi, \quad \mathscr{O}_{\mu \nu}^{5, D}=-\frac{\mathrm{i}}{2} \bar{\psi} \gamma_{\mu} \gamma_{5} \stackrel{\leftrightarrow}{D_{\nu}} \psi, \\
\mathscr{O}_{\mu \nu \omega}^{T, D}=-\frac{\mathrm{i}}{2} \bar{\psi}\left[\gamma_{\mu}, \gamma_{v}\right] \overleftrightarrow{D}_{\omega} \psi, \quad \mathscr{O}_{\mu \nu \omega}^{T, \partial}=-\frac{\mathrm{i}}{2} \partial_{\omega}\left(\bar{\psi}\left[\gamma_{\mu}, \gamma_{v}\right] \psi\right) .
\end{gathered}
$$


Operators such as $\mathscr{O}_{\mu \nu \omega}^{T, D}$ are of interest for tensor GPDs as well as for transversity and we call them transversity operators. For non-chiral fermions, operators (2.4) contribute as lower-dimensional operators to mixing in certain representations which determine second moments of GPDs.

As operators with two derivatives we consider

$$
\begin{aligned}
& \mathscr{O}_{\mu \nu \omega}^{D D}=-\frac{1}{4} \bar{\psi} \gamma_{\mu} \stackrel{\leftrightarrow}{D_{\nu}} \stackrel{\leftrightarrow}{D}_{\omega} \psi, \quad \mathscr{O}_{\mu \nu \omega}^{\partial D}=-\frac{1}{4} \partial_{\nu}\left(\bar{\psi} \gamma_{\mu} \stackrel{\leftrightarrow}{D}_{\omega} \psi\right), \mathscr{O}_{\mu \nu \omega}^{\partial \partial}=-\frac{1}{4} \partial_{\nu} \partial_{\omega}\left(\bar{\psi} \gamma_{\mu} \psi\right), \\
& \mathscr{O}_{\mu \nu \omega}^{5, D D}=-\frac{1}{4} \bar{\psi} \gamma_{\mu} \gamma_{5} \stackrel{\leftrightarrow}{D}_{\nu} \stackrel{\leftrightarrow}{D_{\omega}} \psi, \quad \mathscr{O}_{\mu \nu \omega}^{5, \partial D}=-\frac{1}{4} \partial_{\nu}\left(\bar{\psi} \gamma_{\mu} \gamma_{5} \stackrel{\leftrightarrow}{D}_{\omega} \psi\right), \mathscr{O}_{\mu \nu \omega}^{5, \partial \partial}=-\frac{1}{4} \partial_{\nu} \partial_{\omega}\left(\bar{\psi} \gamma_{\mu} \gamma_{5} \psi\right)
\end{aligned}
$$

and the transversity operators

$$
\mathscr{O}_{\mu \nu \omega \sigma}^{T, D D}=-\frac{1}{4} \bar{\psi}\left[\gamma_{\mu}, \gamma_{v}\right] \overleftrightarrow{D}_{\omega} \stackrel{\leftrightarrow}{D}_{\sigma} \psi, \quad \mathscr{O}_{\mu \nu \omega \sigma}^{T, \partial \partial}=-\frac{1}{4} \partial_{\omega} \partial_{\sigma}\left(\bar{\psi}\left[\gamma_{\mu}, \gamma_{v}\right] \psi\right)
$$

On the lattice the operators are classified according to the irreducible representations $\tau_{k}^{(l)}$ of the hypercubic group H(4) (for the notation see, e.g., Ref. [9]). Here $l$ denotes the dimension of the representation and $k$ labels inequivalent representations of the same dimension. In addition our operators will be chosen such that they have definite charge conjugation parity $C$.

To define various index combinations of operators we use the short-hand notations:

$$
\begin{aligned}
\mathscr{O}_{\ldots\left\{v_{1} v_{2}\right\}} & =\frac{1}{2}\left(\mathscr{O}_{\ldots v_{1} v_{2}}+\mathscr{O}_{\ldots v_{2} v_{1}}\right), \\
\mathscr{O}_{\left\{v_{1} v_{2} v_{3}\right\}} & =\frac{1}{6}\left(\mathscr{O}_{v_{1} v_{2} v_{3}}+\mathscr{O}_{v_{1} v_{3} v_{2}}+\mathscr{O}_{v_{2} v_{1} v_{3}}+\mathscr{O}_{v_{2} v_{3} v_{1}}+\mathscr{O}_{v_{3} v_{1} v_{2}}+\mathscr{O}_{v_{3} v_{2} v_{1}}\right), \\
\mathscr{O}_{\left\|v_{1} v_{2} v_{3}\right\|} & =\mathscr{O}_{v_{1} v_{2} v_{3}}-\mathscr{O}_{v_{1} v_{3} v_{2}}+\mathscr{O}_{v_{3} v_{1} v_{2}}-\mathscr{O}_{v_{3} v_{2} v_{1}}-2 \mathscr{O}_{v_{2} v_{3} v_{1}}+2 \mathscr{O}_{v_{2} v_{1} v_{3}}, \\
\mathscr{O}_{\left\langle\left\langle v_{1} v_{2} v_{3}\right\rangle\right\rangle} & =\mathscr{O}_{v_{1} v_{2} v_{3}}+\mathscr{O}_{v_{1} v_{3} v_{2}}-\mathscr{O}_{v_{3} v_{1} v_{2}}-\mathscr{O}_{v_{3} v_{2} v_{1}} .
\end{aligned}
$$

For the first moments we choose the following representations and operators:

\begin{tabular}{lcc} 
Operator & Representation & $C$ \\
\hline $\mathscr{O}_{\{14\}}^{D}$ & $\tau_{3}^{(6)}$ & +1 \\
$\mathscr{O}_{44}^{D}-\frac{1}{3}\left(\mathscr{O}_{11}^{D}+\mathscr{O}_{22}^{D}+\mathscr{O}_{33}^{D}\right)$ & $\tau_{1}^{(3)}$ & +1 \\
$\mathscr{O}_{\{14\}}^{5, D}$ & $\tau_{4}^{(6)}$ & -1 \\
$\mathscr{O}_{44}^{5, D}-\frac{1}{3}\left(\mathscr{O}_{11}^{5, D}+\mathscr{O}_{22}^{5, D}+\mathscr{O}_{33}^{5, D}\right)$ & $\tau_{4}^{(3)}$ & -1 \\
$\mathscr{O}_{\langle\langle 124\rangle\rangle}^{T, D}$ & $\tau_{2}^{(8)}$ & +1 \\
$\mathscr{O}_{\langle\langle 122\rangle\rangle}^{T, D}-\mathscr{O}_{\langle\langle 133\rangle\rangle}^{T, D}$ & $\tau_{1}^{(8)}$ & +1
\end{tabular}

All operators in (2.8) are multiplicatively renormalisable. These representations exhaust all possibilities for the twist-2 sector in the continuum. 
In these proceedings we present results for the following sets of mixing twist- 2 operators which are related to the second moments of GPDs in the unpolarised case:

$$
\begin{aligned}
& \frac{\tau_{2}^{(4)}, C=-1}{\tau_{1}^{(8)}, C=-1}: \quad \mathscr{O}_{\{124\}}^{D D}, \mathscr{O}_{\{124\}}^{\partial \partial} \\
& \mathscr{O}_{1}=\mathscr{O}_{\{114\}}^{D D}-\frac{1}{2}\left(\mathscr{O}_{\{224\}}^{D D}+\mathscr{O}_{\{334\}}^{D D}\right), \mathscr{O}_{2}=\mathscr{O}_{\{114\}}^{\partial \partial}-\frac{1}{2}\left(\mathscr{O}_{\{224\}}^{\partial \partial}+\mathscr{O}_{\{334\}}^{\partial \partial}\right), \\
& \mathscr{O}_{3}=\mathscr{O}_{\langle\langle 114\rangle\rangle}^{D D}-\frac{1}{2}\left(\mathscr{O}_{\langle\langle 224\rangle\rangle}^{D D}+\mathscr{O}_{\langle\langle 334\rangle\rangle}^{D D}\right), \mathscr{O}_{4}=\mathscr{O}_{\langle\langle 114\rangle\rangle}^{\partial \partial}-\frac{1}{2}\left(\mathscr{O}_{\langle\langle 224\rangle\rangle}^{\partial \partial}+\mathscr{O}_{\langle\langle 334\rangle\rangle}^{\partial \partial}\right), \\
& \mathscr{O}_{5}=\mathscr{O}_{\|\mid 213\|}^{5, \partial D}, \mathscr{O}_{6}=\mathscr{O}_{\langle\langle 213\rangle\rangle}^{5, \partial D}, \mathscr{O}_{7}=\mathscr{O}_{\|213\|}^{5, D D}, \mathscr{O}_{8}=\mathscr{O}_{411}^{T, \partial}-\frac{1}{2}\left(\mathscr{O}_{422}^{T, \partial}+\mathscr{O}_{433}^{T, \partial}\right) .
\end{aligned}
$$

In the polarised case we consider

$$
\begin{aligned}
& \frac{\tau_{3}^{(4)}, C=+1}{\frac{\tau_{2}^{(8)}, C=+1}{2}:}: \mathscr{O}_{\{124\}}^{5, D D}, \mathscr{O}_{\{124\}}^{5, \partial \partial} \\
& \mathscr{O}_{1}^{5}=\mathscr{O}_{\{114\}}^{5, D D}-\frac{1}{2}\left(\mathscr{O}_{\{224\}}^{5, D D}+\mathscr{O}_{\{334\}}^{5, D D}\right), \mathscr{O}_{2}^{5}=\mathscr{O}_{\{114\}}^{5, \partial \partial}-\frac{1}{2}\left(\mathscr{O}_{\{224\}}^{5, \partial \partial}+\mathscr{O}_{\{334\}}^{5, \partial \partial}\right), \\
& \mathscr{O}_{3}^{5}=\mathscr{O}_{\langle\langle 114\rangle\rangle}^{5, D D}-\frac{1}{2}\left(\mathscr{O}_{\langle\langle 224\rangle\rangle}^{5, D D}+\mathscr{O}_{\langle\langle 334\rangle\rangle}^{5, D D}\right), \mathscr{O}_{4}^{5}=\mathscr{O}_{\langle\langle 114\rangle\rangle}^{5, \partial \partial}-\frac{1}{2}\left(\mathscr{O}_{\langle\langle 224\rangle\rangle}^{5, \partial \partial}+\mathscr{O}_{\langle\langle 334\rangle\rangle}^{5, \partial \partial}\right), \\
& \mathscr{O}_{5}^{5}=\mathscr{O}_{\|213\|}^{\partial D}, \mathscr{O}_{6}^{5}=\mathscr{O}_{\langle\langle 213\rangle\rangle}^{\partial D}, \mathscr{O}_{7}^{5}=\mathscr{O}_{\|213\|}^{D D}, \mathscr{O}_{8}^{5}=\mathscr{O}_{123}^{T, D}-2 \mathscr{O}_{231}^{T, D}-\mathscr{O}_{132}^{T, D} .
\end{aligned}
$$

\section{Results}

We calculate the matrix elements in one-loop lattice perturbation theory in the infinite volume limit following Kawai et al. [10]. The renormalisation factors for operators related to the first moments of GPDs are the same as in the forward case and known for the popular representations.

In the case of the second moments of GPDs and distribution amplitudes we present the renormalisation matrices in the form

$$
Z_{i k}(a \mu)=\delta_{i k}-\frac{g_{R}^{2} C_{F}}{16 \pi^{2}}\left(\gamma_{i k} \ln \left(a^{2} \mu^{2}\right)+B_{i k}\right)
$$

where $\gamma$ denotes the matrix of anomalous dimensions and $B$ is the matrix of finite one-loop contributions. Below, the numbers in boldface indicate results known from the forward case.

In the representation (2.9) two operators mix in the non-forward case leading to

$$
\begin{gathered}
\gamma=\left(\begin{array}{cc}
\frac{25}{6} & -\frac{5}{6} \\
0 & 0
\end{array}\right), \quad B^{\text {overlap }}=\left(\begin{array}{cc}
-\mathbf{4 7 . 4 4 4 1}-0.95719 \\
0 & -17.418
\end{array}\right), \\
B^{\text {clover }}=\left(\begin{array}{cr}
-\mathbf{1 1 . 5 6 3}+2.898 c_{s w}-0.984 c_{s w}^{2} & 0.024-0.255 c_{s w}-0.016 c_{s w}^{2} \\
0 & 20.618+4.746 c_{s w}-0.543 c_{s w}^{2}
\end{array}\right) .
\end{gathered}
$$

The results do not depend on the particular choice of the covariant derivative in the non-forward case. 
For the representation (2.10) six operators of the same dimension mix (in the one-loop approximation the contribution of $\mathscr{O}_{7}$ is absent). The finite contributions for clover fermions $B^{\text {clover }}=$ $B^{(0)}+c_{s w} B^{(1)}+c_{s w}^{2} B^{(2)}$ can be found in [5] for the two different derivatives. For the choice (I) of the derivative we find for overlap fermions

$$
\begin{gathered}
\gamma=\left(\begin{array}{rrrccc}
\frac{\mathbf{2 5}}{\mathbf{6}} & -\frac{5}{6} & 0 & 0 & 0 & 0 \\
0 & 0 & 0 & 0 & 0 & 0 \\
0 & 0 & \frac{7}{6} & -\frac{5}{6} & 1 & -\frac{3}{2} \\
0 & 0 & 0 & 0 & 0 & 0 \\
0 & 0 & 0 & 0 & 2 & -2 \\
0 & 0 & 0 & 0 & -\frac{2}{3} & \frac{2}{3}
\end{array}\right) \\
B^{\text {overlap }}=\left(\begin{array}{rrrrrrr}
-\mathbf{4 8 . 1 0 8 9} & -3.43393 & \mathbf{0 . 5 3 7 9 5} & 1.33526 & 0.03132 & 0.64459 \\
0 & -17.4180 & 0 & 0 & 0 & 0 \\
\mathbf{3 . 3 1 6 0 2} & 20.2671 & -\mathbf{4 6 . 8 4 1 6} & -10.9597 & -3.52389 & 1.79504 \\
0 & 0 & 0 & -17.41796 & 0 & 0 \\
0 & -12.2181 & 0 & 0 & -34.1678 & 1.91865 \\
0 & -12.2181 & 0 & 0 & 0.6396 & -32.8888
\end{array}\right)
\end{gathered}
$$

Using clover fermions and the representation (2.10), there is a dangerous mixing between the operator $\mathscr{O}_{1}$ and the operator $\mathscr{O}_{8}$ which is one dimension lower than $\mathscr{O}_{1}$ :

$$
\left.\mathscr{O}_{1}^{\text {clover }}\right|_{1 / a-\text { part }}=\frac{g_{R}^{2} C_{F}}{16 \pi^{2}}\left(-0.51771+0.08325 c_{s w}-0.00983 c_{s w}^{2}\right) \frac{1}{a} \mathscr{O}_{8}^{\text {Born }}
$$

This mixing leads to a contribution which diverges like the inverse lattice spacing in the continuum limit. Thus the perturbative calculation of the mixing coefficient is not reliable and the operator $\mathscr{O}_{8}$ has to be subtracted non-perturbatively from $\mathscr{O}_{1}$. Note, however, that $\mathscr{O}_{1}$ and $\mathscr{O}_{8}$ are of opposite chirality.

Using chiral lattice fermions in perturbation theory, the general matrix element of an operator with two covariant derivatives contains also $1 / a$ contributions indicating the potential presence of lower dimensional operators at one-loop level. Choosing a particular irreducible representation such as (2.10), the contributing terms are expected to cancel. This indeed happens in our calculation with overlap fermions and the mixing is absent to a very high accuracy numerically:

$$
\left.\mathscr{O}_{1}^{\text {overlap }}\right|_{1 / a-\text { part }}=0 \text {. }
$$

Using the operators (2.11) and (2.12) (in the polarised case) the one-loop results for the chiral overlap fermions coincide with those given in (3.2) and (3.4), (3.5), respectively.

\section{Summary}

We have considered quark-antiquark operators needed for the computation of the first two moments of GPDs and meson distribution amplitudes within the framework of lattice QCD. In one-loop lattice perturbation theory we have calculated the non-forward quark matrix elements of these operators employing clover improved Wilson fermions and overlap fermions together with 
Wilson's plaquette action for the gauge fields. From the results we have determined the matrices of renormalisation and mixing coefficients in the $\overline{\mathrm{MS}}$-scheme for some sets of operators belonging to particular representations of the hypercubic group.

If there is only mixing between one operator with two covariant derivatives $D$ and one operator with two external derivatives $\partial$ (e.g. for the representation (2.9) with three different Lorentz indices) the mixing coefficient turns out to be rather small. In the case of the representation (2.10) with two equal Lorentz indices - which contains eight potentially mixing operators - the mixing effects are more severe. As expected, the dangerous mixing with a lower-dimensional operator of opposite chirality in the case of clover or Wilson fermions is absent using overlap fermions. This obviously favours the use of chiral fermions in future simulations related to GPDs and DAs.

Results for a more complete set of representations using overlap fermions will be presented elsewhere [11].

\section{Acknowledgements}

This work is supported by DFG under contract FOR 465 (Forschergruppe Gitter-HadronenPhänomenologie) and by the EU Integrated Infrastructure Initiative Hadron Physics under contract number RII3-CT-2004-506078. We would like to thank A. Schäfer who contributed to part of the results presented here.

\section{References}

[1] M. Diehl, Phys. Rept. 388 (2003) 41 [arXiv:hep-ph/0307382].

[2] V. L. Chernyak and A. R. Zhitnitsky, Phys. Rept. 112 (1984) 173; V. M. Braun, G. P. Korchemsky and D. Müller, Prog. Part. Nucl. Phys. 51 (2003) 311 [arXiv:hep-ph/0306057].

[3] K. Orginos, plenary talk at Lattice06, these proceedings.

[4] M. Göckeler, R. Horsley, H. Perlt, P. E. L. Rakow, A. Schäfer, G. Schierholz and A. Schiller, Nucl. Phys. B 717 (2005) 304 [arXiv:hep-lat/0410009].

[5] M. Göckeler, R. Horsley, H. Perlt, P. E. L. Rakow, A. Schäfer, G. Schierholz and A. Schiller, arXiv:hep-lat/0605002, EPJC to appear.

[6] B. Sheikholeslami and R. Wohlert, Nucl. Phys. B 259 (1985) 572.

[7] H. Neuberger, Phys. Lett. B 417 (1998) 141 [arXiv:hep-lat/9707022]; Phys. Lett. B 427 (1998) 353 [arXiv:hep-lat/9801031].

[8] S. Capitani, Phys. Rept. 382 (2003) 113 [arXiv:hep-lat/0211036].

[9] M. Göckeler, R. Horsley, E.-M. Ilgenfritz, H. Perlt, P. Rakow, G. Schierholz and A. Schiller, Phys. Rev. D 54 (1996) 5705 [arXiv:hep-lat/9602029].

[10] H. Kawai, R. Nakayama and K. Seo, Nucl. Phys. B 189 (1981) 40.

[11] M. Göckeler et al., in preparation. 